\title{
Homosynaptic Long-Term Depression in the Visual Cortex
}

\author{
Alfredo Kirkwood and Mark F. Bear \\ Department of Neuroscience, Brown University, and Institute for Brain and Neural Systems, Providence, Rhode Island \\ 02912
}

\begin{abstract}
We have investigated the characteristics and mechanism of activity-dependent decreases in synaptic effectiveness in visual cortex. Repetitive, low-frequency stimulation (LFS) of either layer IV or the white matter of visual cortical slices was shown to result in a long-term depression (LTD) of intraand extracellularly recorded synaptic responses in layer III. In preparations in which responses to stimulation of two independent pathways could be monitored, LFS of one pathway produced LTD of responses to test stimulation of that input only, showing that this form of LTD is homosynaptic. This form of LTD was dependent on the frequency and/or pattern of conditioning stimulation and on activation of NMDA receptors. Okadaic acid, an inhibitor of protein phosphatases 1 and $2 a$, inhibited LTD, but had no effect on induction of long-term potentiation. In all of these respects, LFS-induced LTD in visual cortex closely resembles what has been recently documented in hippocampus. The combined data support a model in which LTD is triggered by a modest elevation in postsynaptic $\mathrm{Ca}^{2+}$ and activation of protein-serine, threonine phosphatases.
\end{abstract}

[Key words: synaptic plasticity, development, phosphatase, NMDA receptors, neocortex]

In the neocortex, there is ample evidence that there exists a process of activity-dependent depression of synaptic effectiveness. One clear example is the synaptic disconnection of inputs to visual cortex that serve an eye that has been temporarily deprived of vision during early postnatal life (Wiesel, 1982). Other examples are the use-dependent shifts in receptive fields in the adult somatosensory (Merzenich, 1987) and visual (Gilbert and Wiesel, 1992) cortex. Similar experience-dependent shifts in neuronal stimulus selectivity are believed to be a likely basis for cortically dependent learning and memory throughout life (Miller et al., 1991). Thus, a question of considerable interest concerns the principles and underlying mechanisms of synaptic long-term depression (LTD) in neocortex.

According to a theory developed to account for the experience-dependent plasticity of visual cortical response properties, activation of a modifiable excitatory input should lead to an increase or a decrease in synaptic effectiveness depending on whether the coincident activity of the postsynaptic target falls above or below a critical value called the "modification thresh-

Received Aug. 17, 1993; accepted Nov. 15, 1993.

We thank D. Brautigan for many helpful discussions and for generously providing okadaic acid, and $R$. Malenka for sharing his results with us before they were published. This work was supported by the U.S. Office of Naval Research, The National Eye Institute, and the Human Frontiers Science Program.

Correspondence should be addressed to Mark Bear, Department of Neuroscience, Brown University, Box 1953, Providence, RI 02912.

Copyright (C) 1994 Society for Neuroscience $0270-6474 / 94 / 143404-09 \$ 05.00 / 0$ old" (Cooper et al., 1979; Bienenstock et al., 1982). It was proposed that the modification threshold corresponds to the level of NMDA receptor activation that is sufficient to trigger longterm potentiation (LTP) (Bear et al., 1987). From this postulate it is axiomatic that input activity that consistently fails to activate NMDA receptors sufficiently to cause LTP instead should cause ITD of the active synapses.

This idea was recently tested in the CAl region of hippocampus (Dudek and Bear, 1992, 1993). Repetitive activation of the Schaffer collaterals at frequencies of $1-3 \mathrm{~Hz}$ was found to cause a decrease in synaptic effectiveness that persisted without signs of recovery for $>1 \mathrm{hr}$ in vitro. This form of LTD was specific to the stimulated inputs (i.e., it was homosynaptic) and was dependent upon activation of NMDA receptors. Subsequent work showed that this homosynaptic LTD requires for induction an elevation of postsynaptic $\mathrm{Ca}^{2+}$ during the conditioning stimulation (Mulkey and Malenka, 1992). The data therefore support a model in which the state of correlation of pre- and postsynaptic activity in CAl is converted by the voltage-dependent NMDA receptor channel into a graded postsynaptic $\mathrm{Ca}^{2+}$ signal that triggers LTP when it cxcceds some critical valuc (the "modification threshold") and triggers LTD when it falls below this level. Since one of the initial steps in LTP induction is activation of $\mathrm{Ca}^{2+}$-dependent protein kinases (Malenka et al., 1989; Mallinow et al., 1989), this model could be realized if modest elevations of $\mathrm{Ca}^{2+}$ were to activate preferentially protein phosphatases that act on the same substrates (Lisman, 1989). Consistent with this idea, very recent work has shown that inhibition of protein phosphatases 1 and 2 a can block induction of homosynaptic LTD in CA1 (Mulkey et al., 1993).

As compelling as the data are in $\mathrm{CAl}$, the functional role of LTD in this location remains to be determined, and it is uncertain to what extent this model can be applied to synaptic plasticity in the neocortex. For example, NMDA receptor activation appears to inhibit a form of homosynaptic LTD that is triggered by strong stimulation in rat neocortex (Artola et al., 1990; Hirsch and Crepel, 1991). Thus, the question of whether a similar mechanism accounts for homosynaptic LTD in hippocampus and neocortex across species and postnatal ages has remained open.

We recently examined this issue in slices of hippocampus and visual cortex, and found that these distantly related cortical structures could exhibit surprisingly similar forms of synaptic plasticity in vitro if they were studied in a similar fashion (Kirkwood et al., 1993). In particular, we adopted the protocol that was successful in CAl and were able to demonstrate that a similar form of synaptic LTD could be elicited in layer III of visual cortex. The goal of the present investigation was to elaborate on these initial findings and to address the question of mechanism of LTD in visual cortex. Among the new findings 
reported in this article are (1) that unlike LTP, LTD can be evoked either from layer IV or the white matter, (2) that the same number of conditioning pulses can yield LTD or LTP depending on the temporal structure of the conditioning stimulation, and (3) that okadaic acid, a potent inhibitor of protein phosphatases 1 and 2a, inhibits LTD but has no effect on LTP in visual cortex.

\section{Materials and Methods}

The experiments described in this article were performed on slices prepared from the visual cortex of adult Long-Evans rats $(\geq 150 \mathrm{gm})$ or, when indicated, kittens between 5 and 8 weeks of age. Each animal was given an overdose of sodium pentobarbital $(\sim 75 \mathrm{mg} / \mathrm{kg}$, i.p.) and was decapitated soon after the disappearance of any corneal reflexes. The brain was rapidly removed and immersed in ice-cold dissection buffer containing, in mM, $\mathrm{NaCl}, 124 ; \mathrm{KCl}, 5 ; \mathrm{NaH}_{2} \mathrm{PO}_{4}, 1.25 ; \mathrm{MgSO}_{4}, 1 ; \mathrm{CaCl}_{2}$, 2; $\mathrm{NaHCO}_{3}, 26$; dextrose, 10; and kynurenate, 1. A block of visual cortex was removed and sectioned in the coronal plane into $0.4-\mathrm{mm}$ thick slices using a Microslicer (DTK 1000, Ted Pella, Inc., Redding, CA). These slices were collected in ice-cold dissection buffer and gently transferred to an interface slice chamber (Medical Systems Corp., Greenvalc, NY). Here, the slices were maintained in an atmosphere of humidified $95 \% \mathrm{O}_{2}$ and $5 \% \mathrm{CO}_{2}$, and superfused with $35^{\circ} \mathrm{C}$ artificial cerebrospinal fluid (ACSF) at a rate of $1 \mathrm{ml} / \mathrm{min}$. The ACSF was saturated with $95 \% \mathrm{O}_{2}$ and $5 \% \mathrm{CO}_{2}$, and had the same composition as the dissection buffer except that kynurenate was omitted. Kynurenate was included in the dissection buffer to prevent any toxicity caused by excitatory amino acid release from the tissue during cutting. Slices were left undisturbed for $\geq 1 \mathrm{hr}$ before beginning an experiment; during this equilibration period in the slice chamber, any traces of kynurenate were washed away.

Microelectrodes were filled with $1 \mathrm{M} \mathrm{NaCl}(1-2 \mathrm{M} \Omega)$ for extracellular recording, or $3 \mathrm{M} \mathrm{K}$-acetate $(80-120 \mathrm{M} \Omega$ ) for intracellular recording. Only cells with resting membrane potentials more negative than -70 $\mathrm{mV}$ and input resistances greater than $20 \mathrm{M} \Omega$ were studied. Synaptic responses were cvoked with $0.02 \mathrm{mscc}$ pulses of 10-200 $\mu \mathrm{A}$ amplitude delivered using a bipolar stimulating electrode (outside diameter, 200 $\mu \mathrm{m}$; FHC 16-60-3). In every experiment, a full input-output curve was generated. Baseline responses were obtained at $0.07-0.03 \mathrm{~Hz}$ using a stimulation intensity that yielded a half-maximal field potential response or an intracellular response that was two-thirds what was necessary to evoke an orthodromic action potential. Stimulation was applied either at the border of white matter and layer VI, or at a site in the middle of the cortex (600-800 $\mu \mathrm{m}$ from the pia) that corresponds mainly to cortical layer IV and upper layer V. To induce LTD, 200900 pulses were delivered at $1 \mathrm{~Hz}$ at the same stimulation intensity used for baseline. To induce LTP, 2-5 episodes of theta burst stimulation (TBS) were delivered at $0.1 \mathrm{~Hz}$. TBS consists of 10-13 stimulus trains delivered at 5-7 Hz; each train consists of four pulses at $100 \mathrm{~Hz}$. The total number of pulses delivered during TBS ranged from 80 to 250 .

Synaptic responses were digitized at $20 \mathrm{kHz}$ and stored on a computer using EXPERIMENTER's WORKBENCH (BrainWave Systems Inc., Boulder, $\mathrm{CO}$ ). The initial slope and amplitude of the intracellular excitatory postsynaptic potential (EPSP), and the amplitude of the field potential were extracted as measures of the magnitude of the cellular and population synaptic responses, respectively.

The only experiments used for analysis were those in which the initial baseline period showed no drift in the response magnitude. Averages were calculated as follows: (1) the response magnitude data for each experiment were expressed as percentages of the preconditioning baseline average, (2) the time scale in each experiment was converted to time from the onset of conditioning, and (3) the time-matched, normalized data were averaged across experiments and expressed as the means $( \pm \mathrm{SEM})$. A $t$ test was used for the statistical comparison of the average baseline response magnitude with the response magnitude measured $20 \mathrm{~min}$ after conditioning stimulation. $P \leq 0.05$ was considered significant.

\section{Results}

Low-frequency stimulation induces LTD of synaptic responses in layer $I I I$

Stimulation typically was applied at a site in the middle of the cortical thickness $(600-800 \mu \mathrm{m}$ from the pia) that corresponds mainly to layer IV, and responses were recorded in layer III. Stimulation at this site activates direct projections of layer IV cells to layer III, and ascending intracortical and corticocortical axons that pass through layer IV en route to layer III. The response to layer IV stimulation typically was monitored using the negative field potential, which reflects the amplitude of an excitatory synaptic current sink.

The effects of 900 pulses delivered to layer IV at $1 \mathrm{~Hz}$ on the layer III field potential amplitude are shown in Figure $1 A(n=$ 7). This low-frequency conditioning stimulation caused a depression of the amplitude of the synaptic response in laycr III that persisted without signs of recovery. Although we usually studied LTD using field potentials evoked from layer IV, in some cases simultaneous intracellular recordings were made from layer III pyramidal cells. A depression of the field potential amplitude always correlated with a decrease in the amplitude and initial slope of the intracellular EPSP. Examples are shown in Figure $1, C$ and $D$.

Our choice of a stimulation site in layer IV was prompted by earlier work that showed that LTP of synaptic responses could be evoked reliably by high-frequency stimulation of this layer, but not of the white matter (Kirkwood et al., 1993). In contrast, we found that low-frequency stimulation (LFS) of the white matter evoked LTD with a magnitude and probability that is comparable to that evoked from layer IV (Fig. 1B).

\section{LFS-induced LTD is input specific}

The LTD following LFS may reflect a generalized heterosynaptic depression of synaptic responses in layer III or, like CAI, it may be homosynaptic. To distinguish these possibilities we sought to isolate two independent paths that converge onto the same postsynaptic targets. If the LTD were homosynaptic, then conditioning stimulation of one path should depress only the response to test stimulation of that path.

We attempted to isolate separate inputs to layer III using two approaches. The first method was to make a radial cut in the slice that extended from the white matter through layer IV, and to then stimulate layer IV on either side of the cut as the layer III field potential was monitored (Fig. $2 A$ ). Field potential responses to stimulation of the two sites were matched for amplitude and showed summation that is expected from independent inputs. Using this approach, we found that LFS of layer IV on one side of the cut produced depression of test stimuli only on that side of the cut. Results from one experiment are presented in Figure 2, $B$ and $C$; average data from five experiments are presented in Figure $2 D$.

The second approach was to place a second stimulating electrode lateral to the recording site in layer III. To ensure that stimulation of this site would not activate re-entrant connections from layer IV, a horizontal cut was made in the slice at the layer III/IV border as shown in Figure $2 E$. Again, the responses from the two sites showed summation confirming pathway independence, and the stimulation intensities were adjusted so that they produced responses of similar size. This method similarly revealed that conditioning stimulation of layer IV depressed the response only to test stimuli of layer IV (see Fig. $4 F$ ). From the combined data, we conclude that the LTD evoked in layer III by layer IV stimulation is input specific and therefore homosynaptic.

\section{$L F S$-induced $L T D$ requires activation of $N M D A$ receptors}

In hippocampus, LTD induction using LFS is prevented by application of the NMDA receptor antagonist 2-amino-5-phos- 

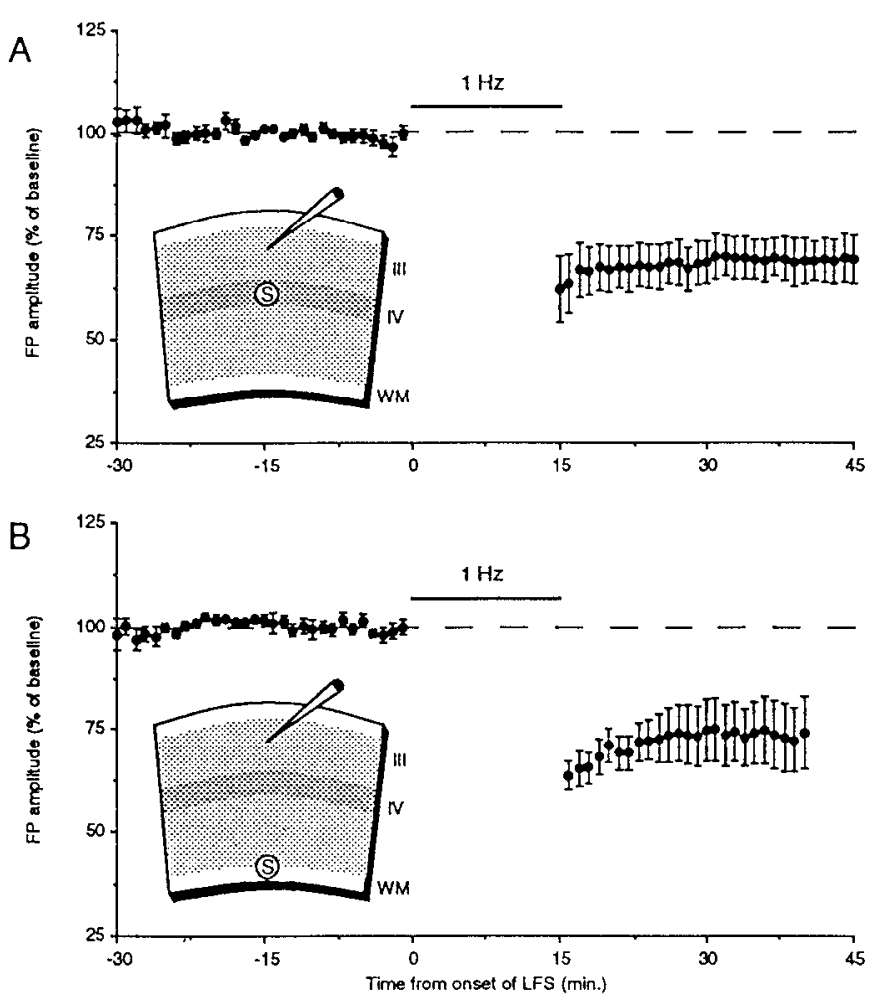

C
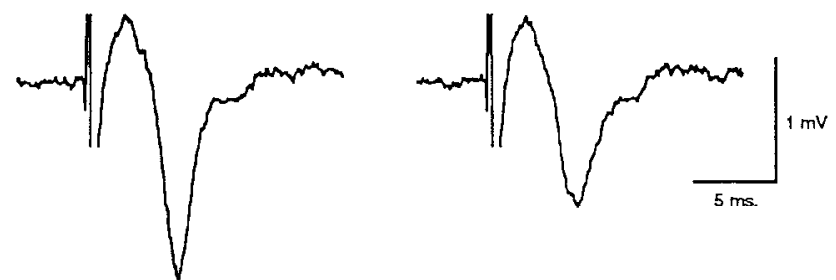

D
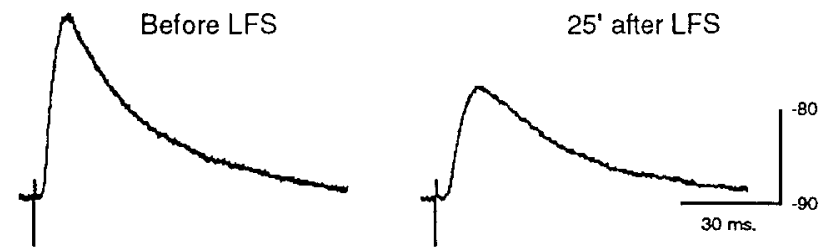

Figure 1. Low-frequency stimulation (LFS) induces LTD of synaptic responses in layer III. $A$, Summary of seven experiments in which field potentials $(F P)$ were evoked in layer III by stimulating a site in the middle of the cortical thickness corresponding mainly to layer IV. The inset is a cartoon of this stimulation-recording arrangement ( $W M$, white matter; $S$, stimulation site). The maximum amplitude of the negative field potential was extracted as a measure of synaptic effectiveness. After a 20 min period in which baseline measurements were made by stimulating at $0.07 \mathrm{~Hz}, 900$ stimuli were applied at $1 \mathrm{~Hz}$. This resulted in LTD of the field potential amplitude. $B$, Summary of five experiments performed identically to those in $A$ except that the white matter was stimulated rather than layer IV (inset). $C$ and $D$, Simultaneously recorded extracellular $(C)$ and intracellular $(D)$ responses in layer III before and after LFS. In this and four similar experiments, a change in the amplitude of the negative field potential always correlated with a change in the amplitude and initial slope of the EPSP.

phonovaleric acid (AP5) (Dudek and Bear, 1992; Mulkey and Malenka, 1992). In visual cortex, in contrast, a form of LTD has been reported that is promoted rather than inhibited by AP5 (Artola et al., 1990; Hirsch and Crepel, 1991). Therefore, it was of interest to assess whether LFS-induced LTD in our neocortical preparation was sensitive to NMDA receptor blockade.

Figure 3 illustrates the results of four experiments in which we attempted to evoke LTD with LFS of layer IV as $100 \mu \mathrm{M}$ D,L-AP5 was bath applied. In none of these experiments were we able to induce LTD; however, in all cases LTD could be elicited following washout of the drug. These data suggest that NMDA receptor activation during the conditioning is necessary for LTD induction in our preparation.

\section{LTD is dependent on the frequency of conditioning stimulation}

The results presented so far indicate that LTD in visual cortex shares two properties with LTP: input specificity and a requirement for activation of NMDA receptors. For NMDA receptors to serve as the trigger for both LTP and LTD, a key variable must be the pattern or amount of activation. In hippocampus this is suggested by the fact that the same number of conditioning pulses delivered at different frequencies yielded different effects: 900 pulses at $1-5 \mathrm{~Hz}$ caused depression, at $10 \mathrm{~Hz}$ there was no net effect, and at $50 \mathrm{~Hz}$ there was a potentiation (Dudek and Bear, 1992). Therefore, it was of interest to determine whether LTD in visual cortex similarly varies as a function of stimulation frequency.

Figure $4 A-C$ shows the average effect on the layer III field potential of 900 pulses delivered at different frequencies to layer IV of rat visual cortex. In comparison with the robust LTD caused by $1 \mathrm{~Hz}$ stimulation, stimulation at $2 \mathrm{~Hz}$ caused only a slight depression of the field potential amplitude, and the effect of $4 \mathrm{~Hz}$ conditioning was negligible. The same experiment was repeated in slices of kitten visual cortex, yielding essentially the same results (Fig. $4 D-F$ ). Thus, as in hippocampus, the effect of LFS on synaptic effectiveness in visual cortex depends on the stimulation frequency, although the precise function relating these variables differs slightly from that previously shown in CA1.

Although in the visual cortex of both species the synaptic depression disappeared when conditioning was given at $4 \mathrm{~Hz}$, the same number of pulses at higher frequencies did not yicld potentiation. We attribute this to possible deleterious effects of prolonged high-frequency stimulation in the neocortical preparations, which tend to be more fragile than hippocampal slices. Therefore, we modified our conditioning protocol to gain further insight into the factors that control the direction and magnitude of synaptic modification in visual cortex. In these experiments, a total of 200 stimuli were given in a "delta burst" pattern (Fig. 5). This conditioning protocol consisted of brief bursts of stimuli at $100 \mathrm{~Hz}$ that were repeated at $1 \mathrm{~Hz}$ (the delta frequency). Although the changes in field potential amplitude that resulted from this type of delta burst stimulation were of small amplitude, presumably due to the small number of pulses, they did vary systematically from depression to potentiation depending on the number of stimuli in each burst (Fig. 5). These results show that the same number of pulses can yield depression or potentiation depending on how the stimuli interact, probably through temporal summation of the EPSPs.

\section{LTD (but not LTP) is inhibited by an inhibitor of protein phosphatases 1 and $2 a$}

Repetitive low-frequency activation of NMDA receptors in CAI may trigger LTD by the selective activation of protein phosphatases (Mulkey et al., 1993). To test for the possible role of protein phosphatases in induction of LTD in visual cortex, we 
A

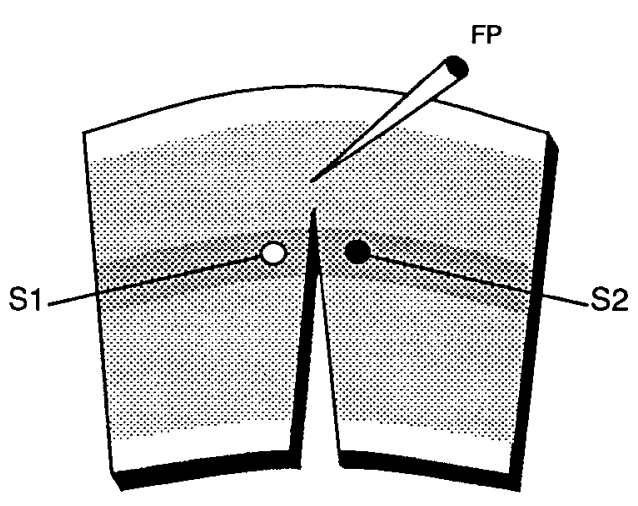

C<smiles>CCCCCCCCC</smiles>
S1

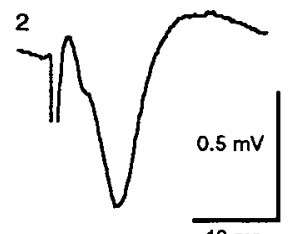

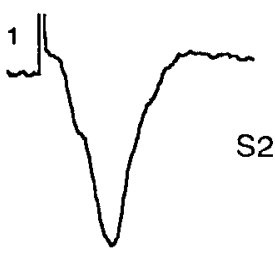

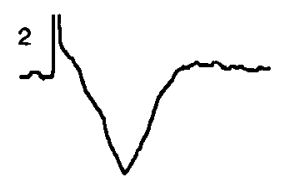

$\mathbf{E}$

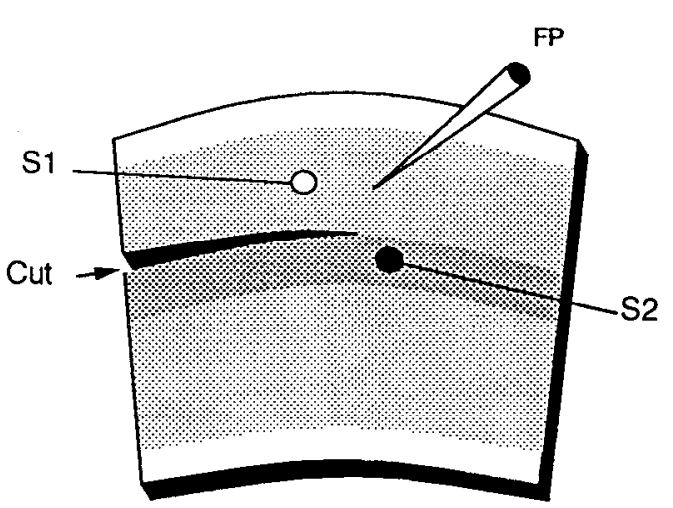

B

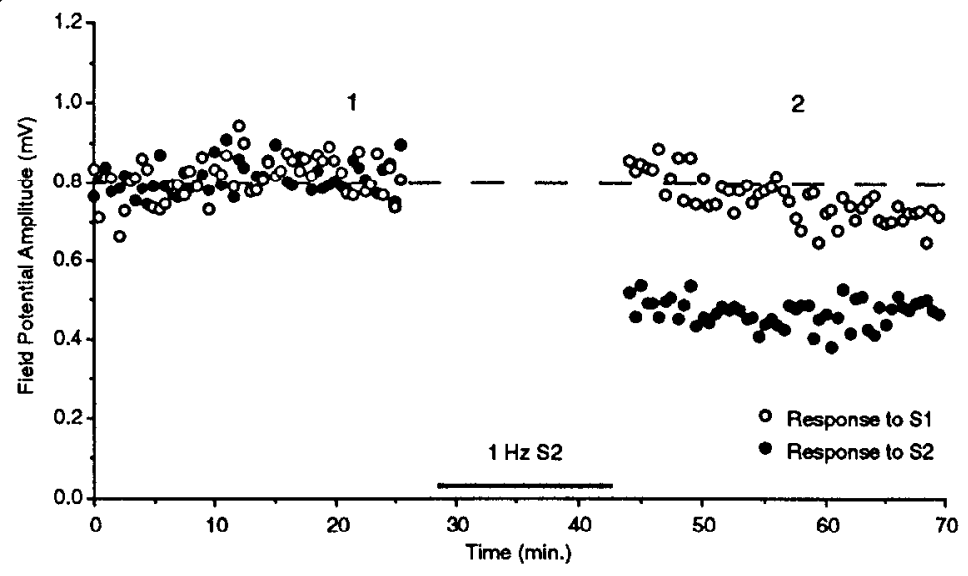

D

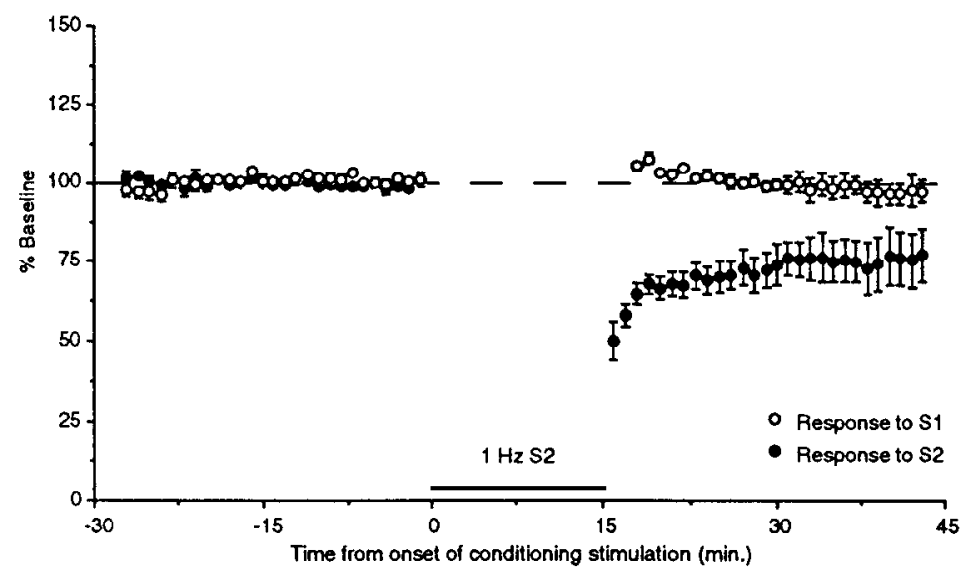

$\mathbf{F}$

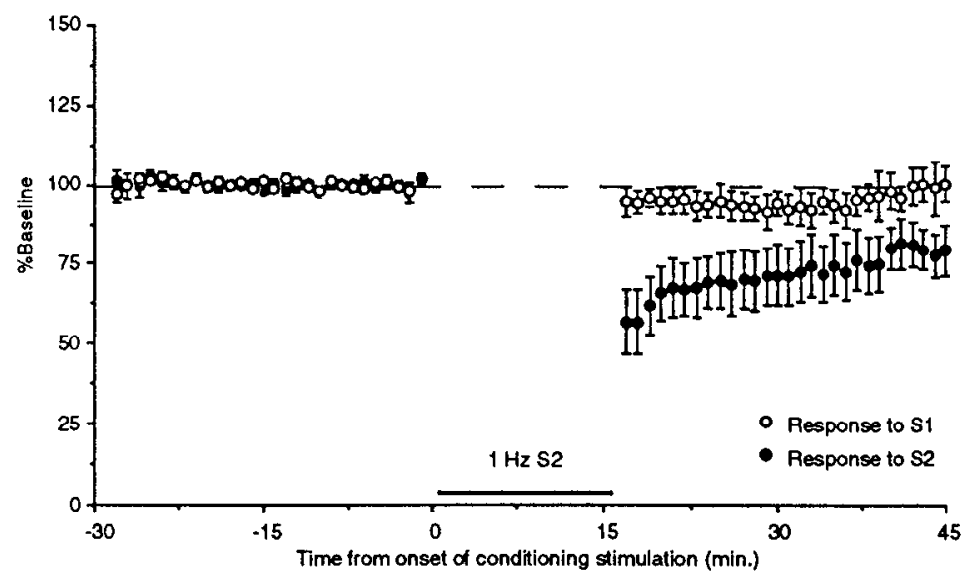

Figure 2. LFS-induced LTD is input specific. A, Cartoon of one stimulation-recording configuration used to assess input specificity. Layer IV was stimulated on either side of a radial cut that extended from the white matter through layer IV. $B$, Record of one experiment using the arrangement in $A$, showing that LFS on one side of the cut produced depression of test stimuli only on that side of the cut. $C$, Field potentials (average of four consecutive traces) recorded in this experiment at the times indicated in $B$. $D$, Average of five experiments performed identically to the individual case in $B$. E , Cartoon of a second stimulation-recording configuration used to assess input specificity. In these cases, a horizontal input from adjacent layer III $(S I)$ was monitored as LTP was attempted from layer IV (S2). $F$. Average of four experiments using the arrangement in $E$ showing that LFS of layer IV produced LTD only in the responses to test stimuli delivered to layer IV. In all experiments designed to test for input specificity, responses from the two inputs showed summation and were matched for size.

applied okadaic acid, a potent and selective inhibitor of protein phosphatases 1 and 2a (Cohen et al., 1990). An example of one experiment is shown in Figure 6. In this case, the responses to two independent inputs from layer IV were monitored. First, in normal buffer, LFS was applied to one path and LTD resulted
(Fig. $6 A$ ). Then $0.5 \mu \mathrm{l}$ of $5 \mu \mathrm{M}$ okadaic acid was applied directly to the slice. This procedure markedly inhibited the induction of LTD by LFS of the second input (Fig. 6B).

On average, topical administration of $0.5-1.0 \mu \mathrm{l}$ of $5 \mu \mathrm{M}$ okadaic acid had little effect on the amplitude of the evoked field 


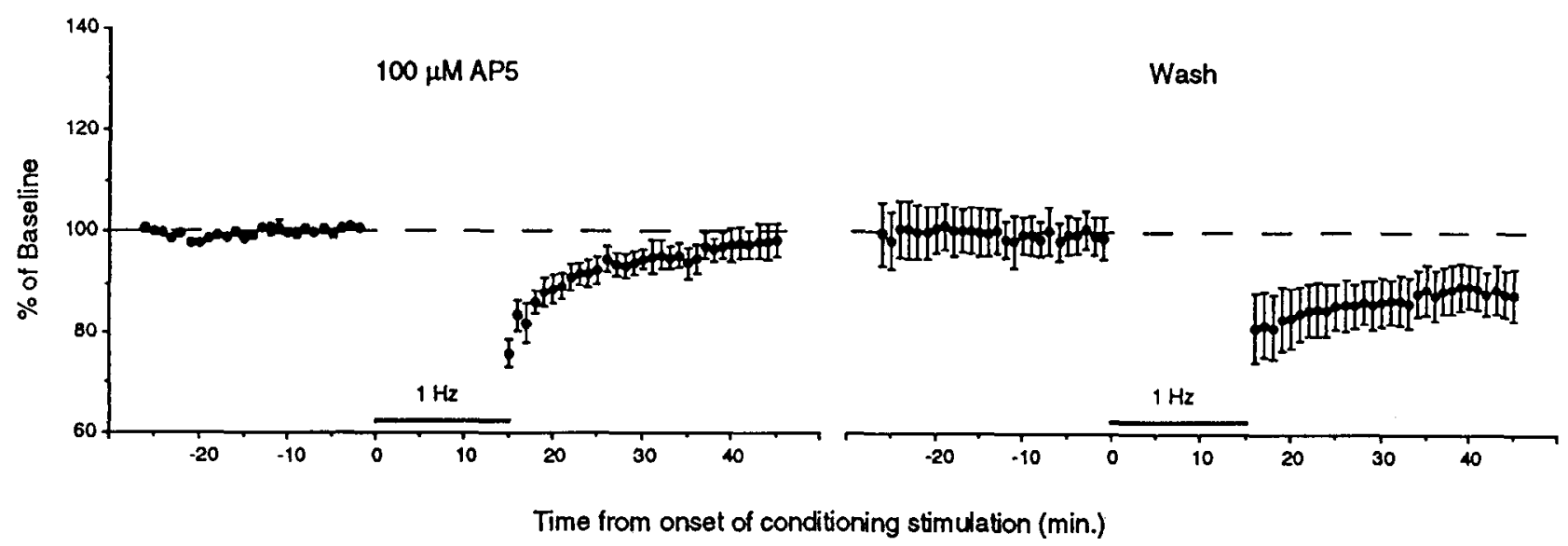

Figure 3. LFS-induced LTD requires activation of NMDA receptors. In the presence of the NMDA receptor antagonist 2-amino-5-phosphonovaleric acid $(A P 5 ; 100 \mu \mathrm{M})$, LFS failed to produce any change in the field potential response to test stimulation. Following washout of the drug, however, LTD was reliably produced $(n=4)$. In both panels of this figure, responses were normalized with respect to the average response during the initial baseline period prior to LFS in AP5. The time that elapsed from the last data point on the left and the first data point on the right varied, but was not greater than $30 \mathrm{~min}$.

Rat
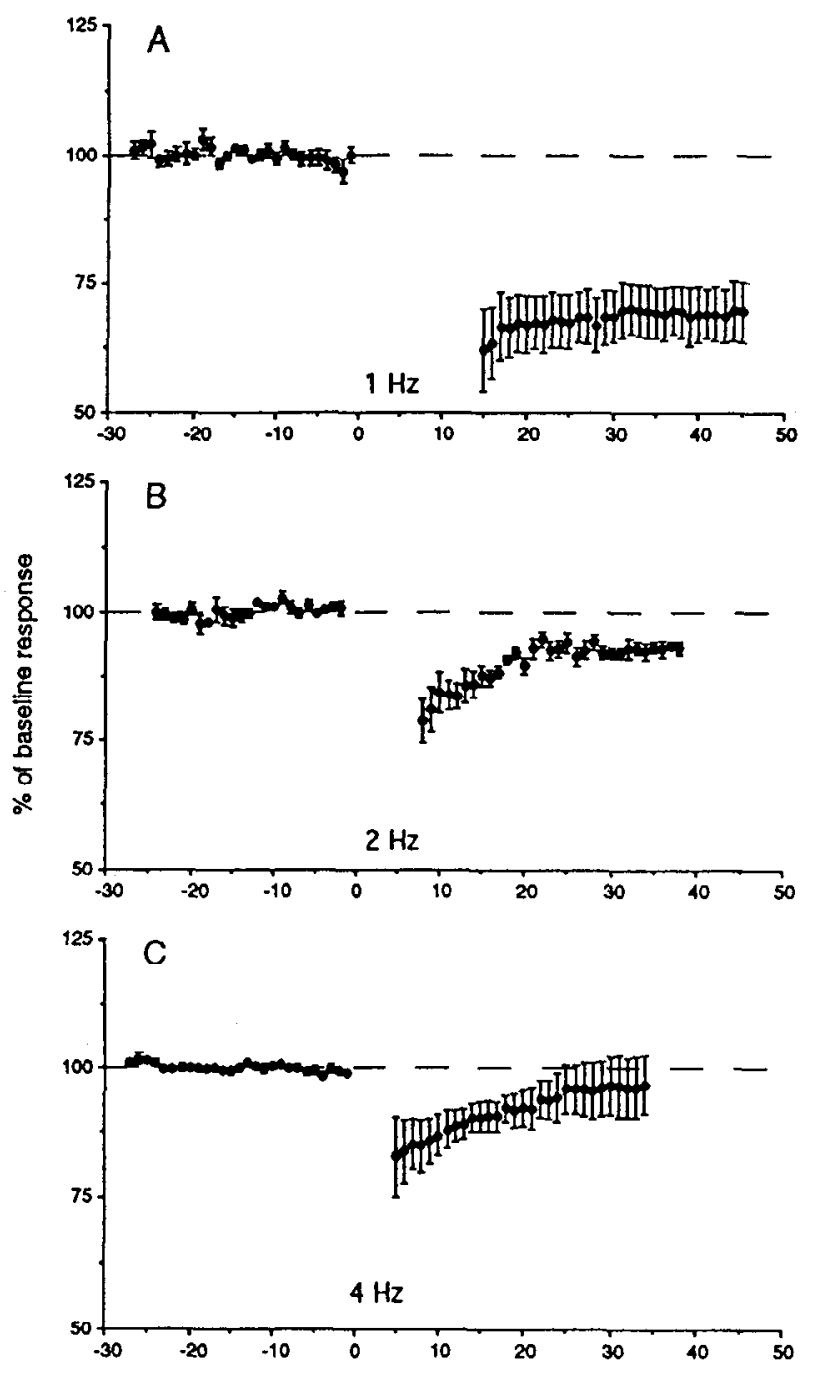

Kitten
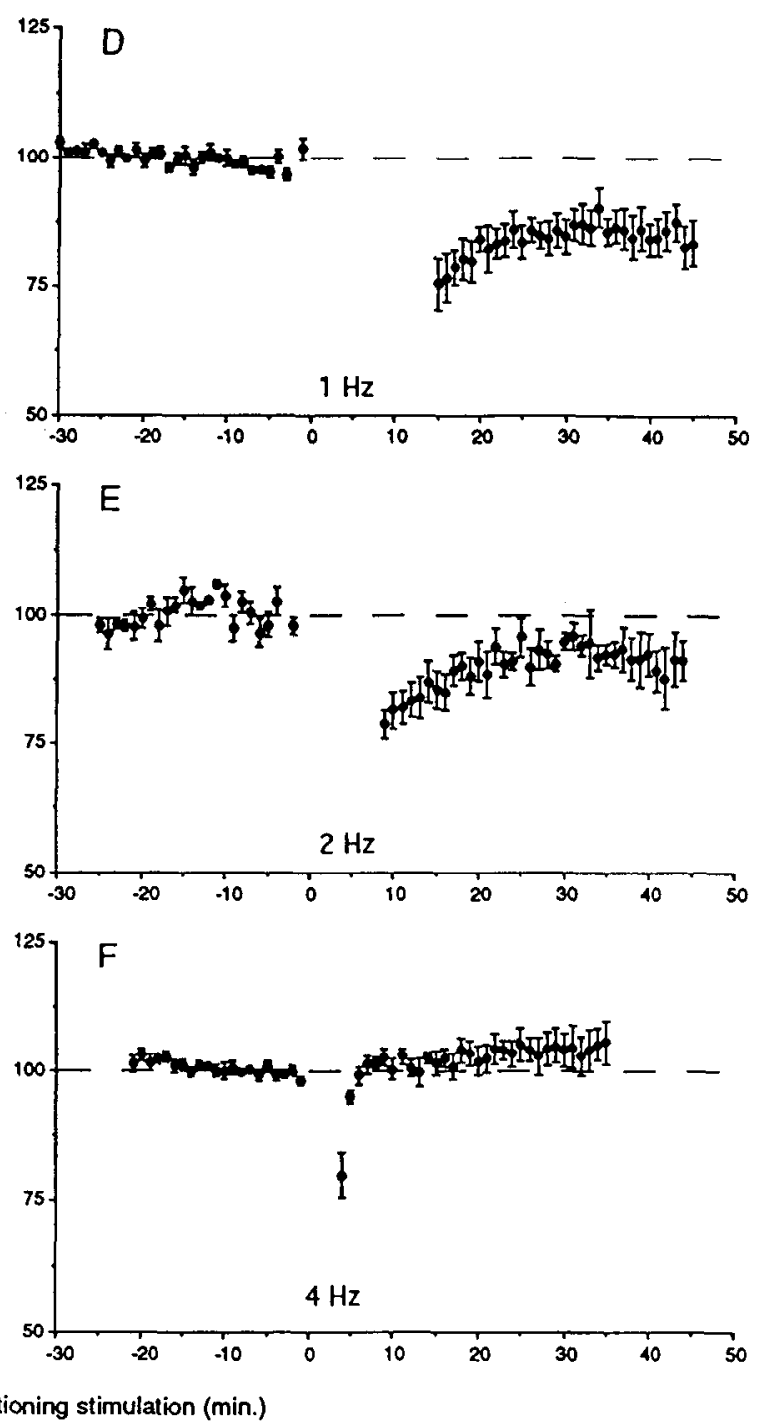

Figure 4. LTD is dependent on the frequency of conditioning stimulation. $A-C$, Average effect of $900 \mathrm{pulses}$ at $1 \mathrm{~Hz}(A ; n=7), 2 \mathrm{~Hz}(B ; n=8)$, and $4 \mathrm{~Hz}(C ; n=6)$ on the field potential response evoked in layer III of adult rat visual cortex by stimulation of layer IV. $D-F$, Average effect of 900 pulses at $1 \mathrm{~Hz}(D ; n=6), 2 \mathrm{~Hz}(E ; n=6)$, and $4 \mathrm{~Hz}(F ; n=5)$ on the field potential response evoked in layer III of 5-7 week old kitten visual cortex by stimulation of layer IV. 


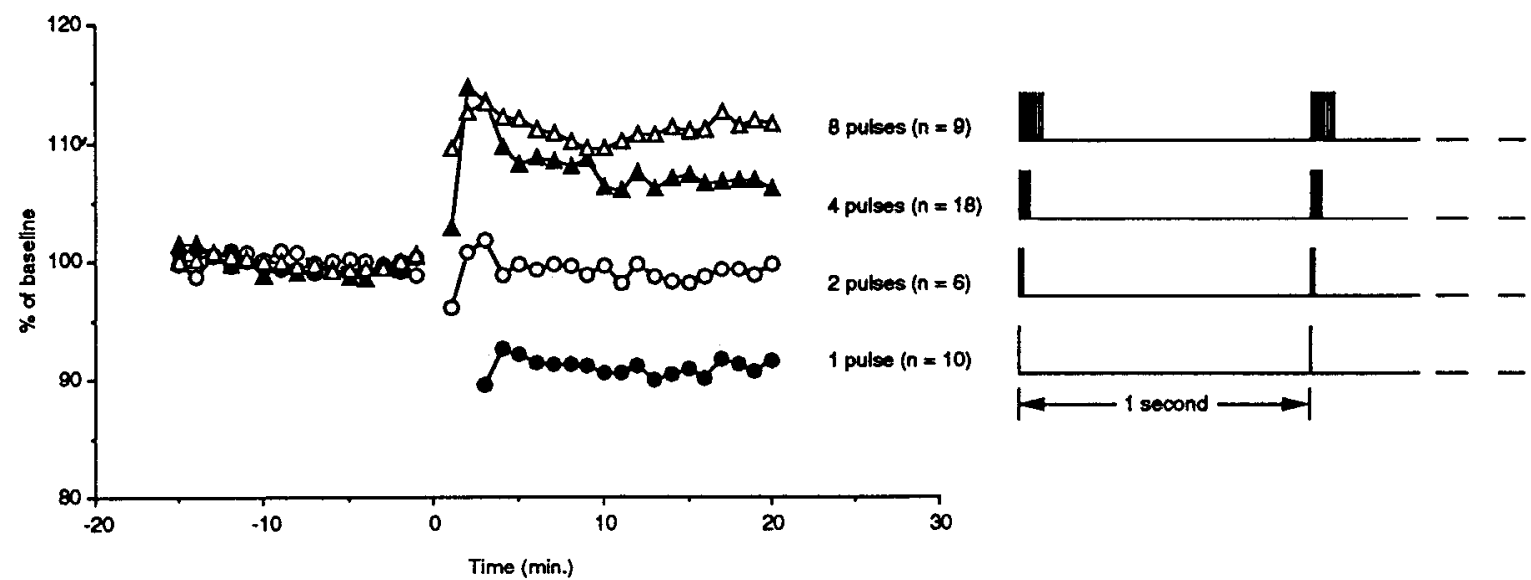

Figure 5. Effects of "delta burst" stimulation on synaptic responses in layer III. A total of 200 conditioning stimuli were delivered to layer IV in the different patterns illustrated on the right. These patterns consisted of bursts of one to eight stimulus pulses that were repeated every second. The burst frequency was $100 \mathrm{~Hz}$. To the left is shown the average change in the synaptic response in layer III following these different types of conditioning stimulation (error bars have been omitted for clarity).

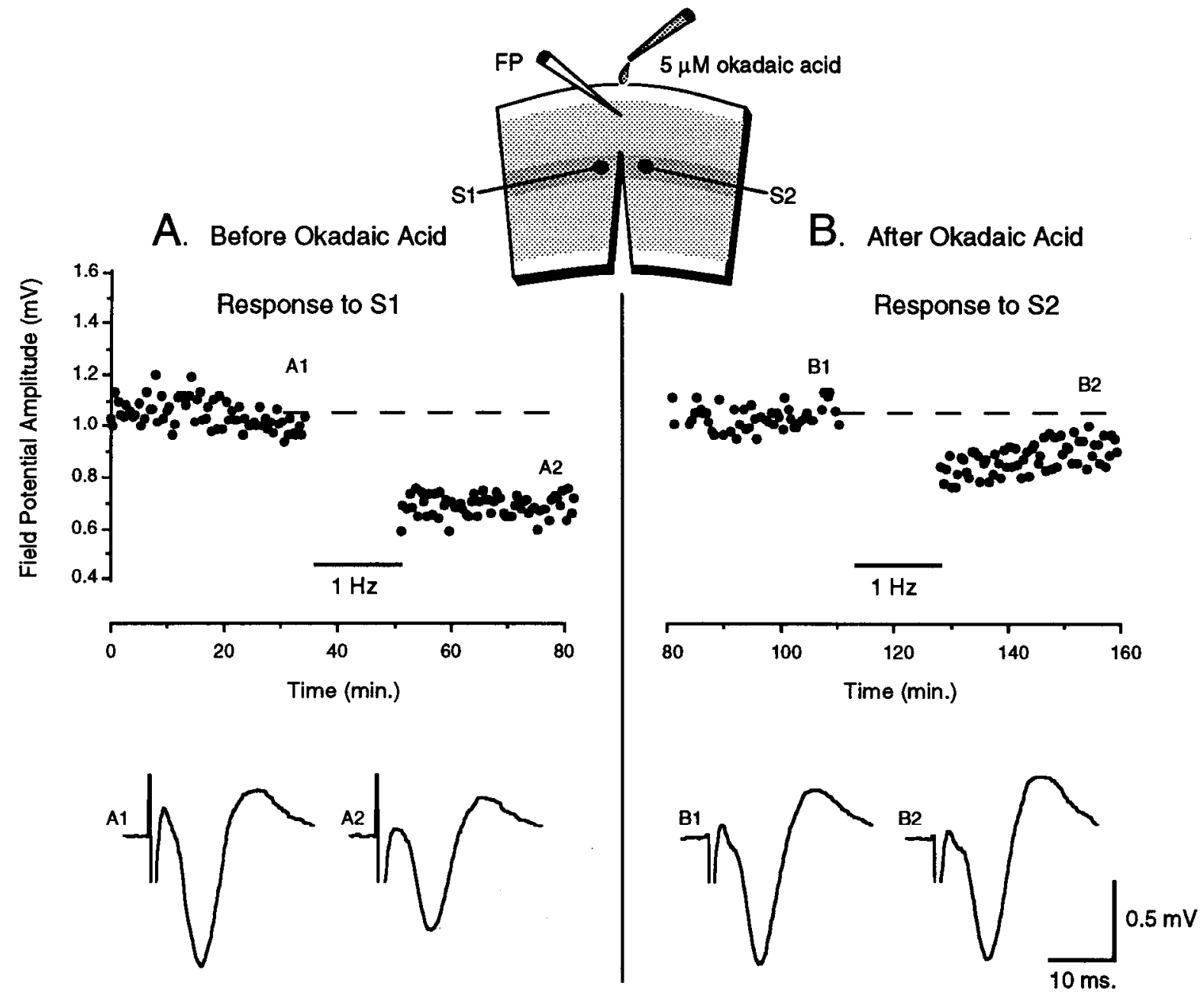

Figure 6. LTD is inhibited by okadaic acid. In this experiment, the responses to stimulation of two pathways $(S 1$ and $S 2)$ were monitored (inset) and these are plotted as a function of time in $A$ and $B$, respectively. $A$, LFS applied to S1 caused LTD as expected, showing that the preparation was viable. Then $0.5 \mu 1$ of $5 \mu \mathrm{M}$ okadaic acid was applied directly to the slice. $B$. Thirty minutes after okadaic acid application, LFS was applied to $\mathrm{S} 2$. This produced only a slight LTD of the response from this pathway. Traces are average field potentials $(n=4)$ collected at the times indicated in $A$ and $B$. 

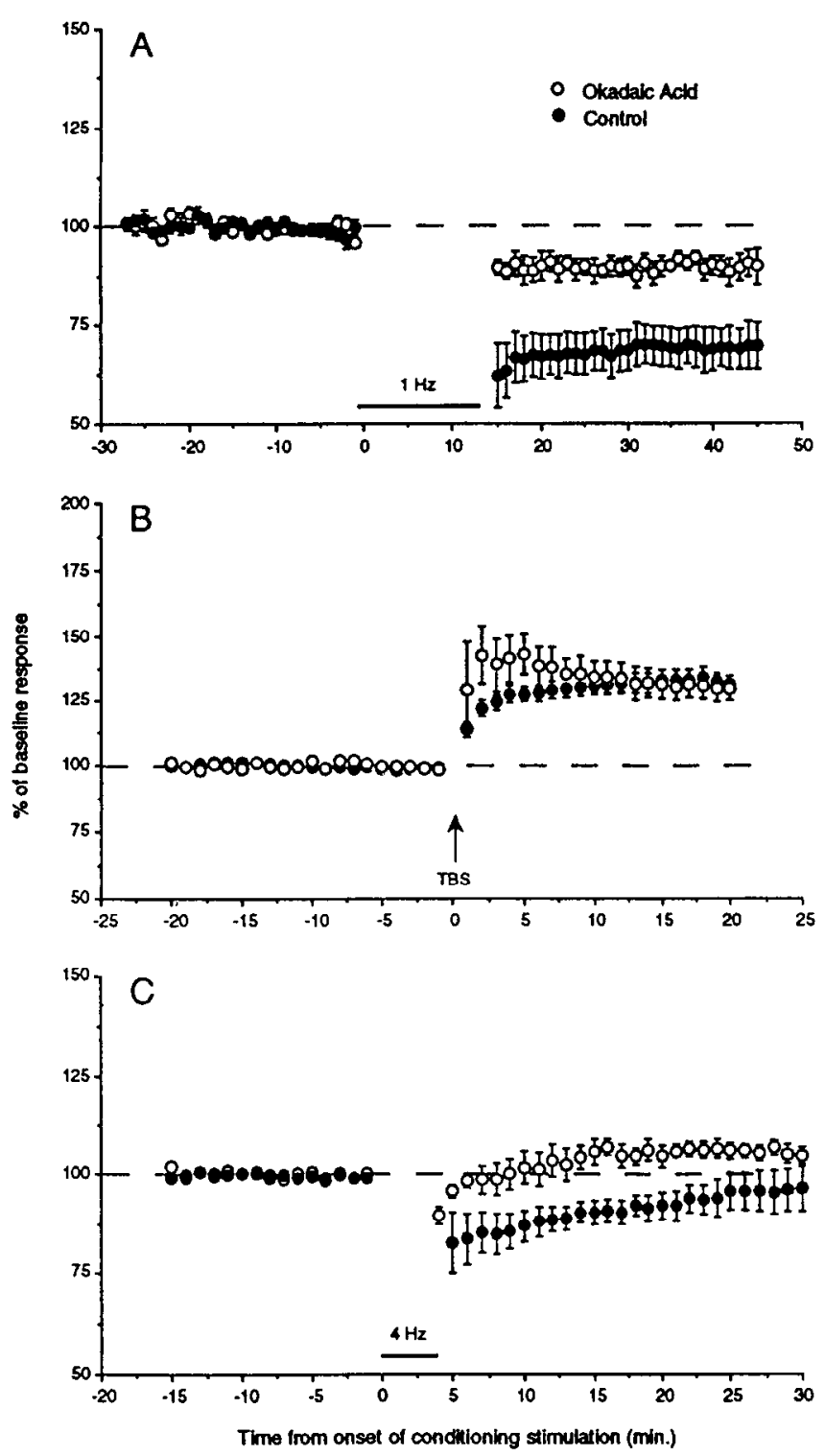

Figure 7. Okadaic acid inhibits LTD but not LTP. A, Following okadaic acid application, LFS produced only a slight decrease in the response (average of seven experiments) compared with control $(n=8)$. This difference is significant $(P<0.001)$. $B$, Okadaic acid had no effect on the induction of LTP by TBS of layer IV (okadaic acid, $n=7$; control, $n=51)$. $C$, In the presence of okadaic acid $(n=4), 4 \mathrm{~Hz}$ stimulation yields a slight but significant $(P<0.001)$ potentiation as compared with control $(n=6)$.

potentials (response $15 \mathrm{~min}$ after okadaic acid administration was $95.6 \pm 9 \%$ of initial baseline response; $n=7$ ), but significantly inhibited the induction of LTD after $1 \mathrm{~Hz}$ conditioning (Fig. 7A). Additional experiments showed that the inhibition of synaptic plasticity by okadaic acid was selective for LTD; induction of LTP by theta burst stimulation (TBS) was unaffected by the drug (Fig. $7 B$ ).

The data are therefore consistent with a model in which LTP is triggered by strong activation of NMDA receptors, a large increase in postsynaptic $\mathrm{Ca}^{2+}$ concentration, and activation of $\mathrm{Ca}^{2+}$-dependent protein kinases, whereas LTD is triggered by weak but prolonged activation of NMDA receptors, a smaller elevation in postsynaptic $\mathrm{Ca}^{2+}$ concentration, and activation of protein phosphatases (Lisman, 1989). If this model is correct, then "neutral" stimulation protocols that activate NMDA receptors but lead to no net change in synaptic effectiveness might cause equal and opposing activation of kinases and phosphatases. We tested this idea by applying 900 pulses at $4 \mathrm{~Hz}$ in the presence of okadaic acid. As shown in Figure $7 C$, in the presence of the drug, this stimulation yielded a slight but significant potentiation of the response as compared with untreated controls.

\section{Discussion}

Here we have followed up on our recent finding that 900 pulses delivered to layer IV at $1 \mathrm{~Hz}$ caused a lasting depression of the field potential recorded in layer III (Kirkwood et al., 1993). In addition to providing complete data on the frequency dependence and AP5 sensitivity of the LTD evoked by LFS, we now document several additional new findings in visual cortex: (1) that repetitive LFS of the white matter as well as layer IV can produce LTD of the synaptic response in layer III, (2) that the input specificity of the LTD applies to radial as well as horizontal inputs to layer III, (3) that a critical variable in inducing LTD is the temporal structure of the conditioning stimulation, probably reflecting the level of postsynaptic activation, and (4) that protein phosphatase activity plays a rolc in induction of LTD, but not LTP.

LTD experiments generally require $>1 \mathrm{hr}$ of stable recordings to obtain a meaningful result; therefore, in most of our experiments we opted to use field potentials to monitor synaptic responses in layer III. We believe that this method is justified for several reasons. First, because care was taken to position the recording electrode at the depth with the maximum negative field potential, we can be confident that changes in the amplitude of this potential reflect changes in a current sink (Mitzdorf, 1985). Second, because this field potential disappears in low $\mathrm{Ca}^{2+}$ (Lee, 1982; Kimura et al., 1989) and is eliminated by broad-spectrum antagonists of excitatory amino acid receptors (Bear et al., 1992), we can be confident that the current sink depends on excitatory synaptic transmission. Third, simultaneous intra- and extracellular recordings show that the peak of the ncgative ficld potential coincides with the maximum rate of rise of the intracellular EPSP, suggesting that the amplitude of this peak reflects a "population EPSC" in layer III (compare Fig. 1). Finally, when simultaneous recordings have been made, LTP and LTD of the layer III field potential have always correlated with LTP and LTD, respectively, of the EPSPs recorded from layer III pyramidal cells (Komatsu et al., 1991; Kirkwood et al., 1993; Kirkwood and Bear, 1994; present results). While uncoupling of the field potential and intracellular responses conceivably could occur if the conditioning caused a persistent change in the resting membrane potentials of layer III neurons, this was not observed in any of our experiments, or in similar studies of LTD in hippocampus (Mulkey and Malenka, 1992).

Although typically we studied LTD in layer III by stimulating a site in the middle of the cortical thickness corresponding mainly to layer IV, we were also successful in inducing LTD in layer III by stimulating the white matter. This contrasts with LTP which, in the absence of GABA antagonists, could not be evoked using TBS when applied to the white matter but could when applied to layer IV (Kirkwood et al., 1992, 1993). To account for that result, we proposed that circuitry within layer IV might act as a type of "plasticity gate" that limits the extent to which the modifiable synapses in layer III can be activated by highfrequency stimulation of the optic radiation (Kirkwood et al., 1993). Our success in inducing LTD from the white matter is 
not inconsistent with this model. If the "gate" acts as a lowpass activity filter, then it is to be expected that LFS-induced LTD would be less affected than TBS-induced LTP.

In neocortex, as in CA1, both LTD and LTP can be blocked by NMDA receptor antagonists. It could be that NMDA receptor activation and resulting $\mathrm{Ca}^{2+}$ entry provides a permissive signal for synaptic plasticity regardless of the sign of the modification. Alternatively, the amount or pattern of NMDA receptor activation could be deciphered postsynaptically to trigger both LTD and LTP. Indeed, when efforts are made to increase gradually the amount of NMDA receptor activation by altering the postsynaptic voltage during conditioning, LTD can be replaced by LTP. This can be shown by giving the same amount of stimulation in patterns designed to increase postsynaptic depolarization through temporal summation of EPSPs (Fig. 5). Most striking, however, is the demonstration that pairing $1 \mathrm{~Hz}$ stimulation with strong intracellular depolarization in both visual cortex (Kirkwood and Bear, 1994) and hippocampus (cf. Colino et al., 1992) results in LTP, whereas $1 \mathrm{~Hz}$ stimulation alone causes LTD.

Previously, evidence has been presented that strong stimulation of neocortex can cause LTD when NMDA receptors are inhibited (Artola et al., 1990; Hirsch and Crepel, 1991; but see Komatsu and Iwakiri, 1992). On the surface, this result appears to be inconsistent with the homosynaptic LTD documented in the present study. However, we note that this NMDA receptorindependent form of LTD also requires an elevation of postsynaptic $\mathrm{Ca}^{2+}$ (Bröcher et al., 1992), as has been shown for the NMDA receptor-dependent form (Mulkey and Malenka, 1992). It is possible that $\mathrm{Ca}^{2+}$ entering through different routes accesses a common biochemical mechanism to trigger LTD.

The physiological model proposed to account for homosynaptic plasticity in visual cortex as a function of postsynaptic membrane potential, in its first conception, posited that NMDA receptor-mediated $\mathrm{Ca}^{2+}$ entry was an exclusive trigger for potentiation (Bear et al., 1987) and that other mechanisms, possibly linked to activation of metabotropic glutamate receptors, served to trigger depression (Bear, 1988; Dudek and Bear, 1989). Although a contribution of metabotropic receptors to LFS-induced LTD remains to be determined, the available data suggest that the information encoded by the pattern or amount of NMDA receptor activation alone may be sufficient to trigger both forms of synaptic plasticity. How can the same signal- $\mathrm{Ca}^{2}$ entry through the NMDA receptor channel-be used to trigger both LTD and LTP? Lisman (1989) showed the feasibility of this type of regulation in a model in which modest elevations of postsynaptic $\mathrm{Ca}^{2+}(<1 \mu \mathrm{M})$ caused LTD by selectively activating protein-serine, threonine phosphatases. Recently, Mulkey et al. (1993) have provided data that support this model in CA1. They report that LFS-induced LTD can be completely blocked by bath application of either okadaic acid or calyculin $\mathrm{A}$, both sclective inhibitors of phosphatases 1 and $2 \mathrm{a}$. In addition, intracellular injection of a third inhibitor, microcystin-L, R, also blocked LTD. Similarly, we have found in our experiments on visual cortex that okadaic acid inhibits induction of homosynaptic LTD. Although inhibition of LTD by okadaic acid was only partial in our experiments, and not complete as reported for CA1, this difference is probably attributable to the different means by which the drug was administered. In the experiments of Mulkey et al. (1993) hippocampal slices were soaked for $>1$ $\mathrm{hr}$ in the inhibitor, whereas we opted for brief, topical application to the visual cortical slices.
Unlike the effects of intracellularly injected $\mathrm{Ca}^{2+}$ buffers and AP5 (Dudek and Bear, 1992; Mulkey and Malenka, 1992), the effects of okadaic acid were selective for LTD; LTP was unaffected (Fig. 7B). This selectivity in the action of okadaic acid makes it unlikely that it inhibits LTD simply by blocking $\mathrm{Ca}^{2+}$ entry through NMDA receptors. And, while a partial block of NMDA receptor conductance might be expected to affect LFSinduced LTD more than TBS-induced LTP, it is difficult to see how this would account for the slight but significant potentiation that followed $4 \mathrm{~Hz}$ conditioning in the presence of okadaic acid (Fig. $7 C$ ). The possibility remains, however, that okadaic acid exerts its effect by enhancing $\mathrm{Ca}^{2+}$ entry through NMDA receptors (e.g., Kelso et al., 1992), thereby shifting the balance from depression to potentiation regardless of the type of conditioning stimulation. We have no data to rule out this possibility, but note that Mulkey et al. (1993) failed to observe any effect of phosphatase inhibitors on isolated NMDA receptormediated conductances in CAl neurons.

The results presented here provide further support for the notion that the elementary forms of synaptic plasticity in CA1 and neocortical layer III are accounted for by common mechanisms (Kirkwood et al., 1993). The powerful implication is that data generated by the study of both systems may contribute to a common foundation for understanding the mechanisms of use-dependent synaptic modification. Indeed, there has already been significant cross-pollination between these different models of cortical plasticity. It was theoretical insights derived from the study of visual cortex that led directly to the current line of investigation on homosynaptic LTD in CAl (i.e., Dudek and Bear, 1992), and it was work on NMDA receptors and LTP in $\mathrm{CA} 1$ that gave rise to current ideas on the physiological basis of "Hebbian" plasticity in the developing visual system (i.e., Bear et al., 1987; Artola and Singer, 1989; Constantine-Paton et al., 1990). One advantage the visual cortex offers is a closer interface between synaptic plasticity and behavior. Thus, it is significant that blockadc of NMDA rcceptors in visual cortex in vivo interferes with both the anatomical (Bear and Coleman, 1990) and physiological (Bear et al., 1990) changes caused by visual deprivation, and that a simple model of visual cortex incorporating homosynaptic LTD and LTP can reproduce the outcome and kinetics of a wide variety of visual deprivation experiments (Clothiaux et al., 1991). We therefore are encouraged to believe that the elementary forms of NMDA receptordependent synaptic plasticity described in vitro contribute importantly to naturally occurring modification in the visual cortex in vivo.

\section{References}

Artola A, Singer W (1989) NMDA receptors and developmental plasticity in visual neocortex. In: The NMDA receptor (Watkins JC, Collingridge GL, eds), p 242. London: Oxford UP.

Artola A, Brocher S, Singer W (1990) Different voltage-dependent thresholds for inducing long-term depression and long-term potentiation in slices of rat visual cortex. Nature 347:69-72.

Bear MF (1988) Involvement of excitatory amino acid receptor mechanisms in the experience-dependent development of visual cortex. In: Frontiers in excitatory amino acid research (Cavalheiro EA, Lehmann J, Turski L, eds), pp 393-401. New York: Liss.

Bear MF, Coleman H (1990) Binocular competition in the control of geniculate cell size depends upon visual cortical $N$-methyl-D-aspartate receptor activation. Proc Natl Acad Sci USA 87:9246-9249.

Bear MF, Cooper LN, Ebner FF (1987) A physiological basis for a thcory of synaptic modification. Science 237:42-48.

Bear MF, Kleinschmidt A, Gu Q, Singer W (1990) Disruption of 
experience-dependent synaptic modifications in striate cortex by infusion of an NMDA receptor antagonist. J Neurosci 10:909-925.

Bear MF, Press WA, Connors BW (1992) Long-term potentiation in slices of kitten visual cortex and the effects of NMDA receptor blockade. J Neurophysiol 67:1-11.

Bienenstock EL, Cooper LN, Munro PW (1982) Theory for the development of neuron selectivity: orientation specificity and binocular interaction in visual cortex. J Neurosci 2:32-48.

Bröcher S, Artola A, Singer W (1992) Intracellular injection of $\mathrm{Ca}(2+)$ chelators blocks induction of long-term depression in rat visual cortex. Proc Natl Acad Sci USA 89:123-127.

Clothiaux EE, Bcar MF, Cooper LN (1991) Synaptic plasticity in visual cortex: comparison of theory with experiment. J Neurophysiol 66: 1785-1804.

Cohen P, Holmes Y, Tsukitani Y (1990) Okadaic acid: a new probe for the study of cellular regulation. Trends Biochem Sci 15:98-102.

Colino A, Huang Y-Y, Malenka RC (1992) Characterization of the integration time for the stabilization of long-term potentiation in area CAl of the hippocampus. J Neurosci 12:180-187.

Constantine-Paton M, Cline HT, Debski E (1990) Patterned activity, synaptic convergence, and the NMDA receptor in developing visual pathways. Annu Rev Neurosci 13:129-154.

Cooper LN, Liberman F, Oja E (1979) A theory for the acquisition and loss of neuron specificity in visual cortex. Biol Cybern 33:9-28.

Dudek SM, Bear MF (1989) A biochemical correlate of the critical period for synaptic modification in the visual cortex. Science 246 : 673-675.

Dudek SM, Bear MF (1992) Homosynaptic long-term depression in area CA1 of hippocampus and the effects of NMDA receptor blockade. Proc Natl Acad Sci USA 89:4363-4367.

Dudek SM, Bear MF (1993) Bidirectional long-term modification of synaptic effectiveness in the adult and immature hippocampus. $J$ Neurosci 13:2910-2918.

Gilbert CD. Wiesel TN (1992) Receptive field dynamics in adult primary visual cortex. Nature 356:150-152.

Hirsch JC, Crepel F (1991) Blockade of NMDA receptors unmasks a long-term depression in synaptic efficacy in rat prefrontal neurons in vitro. Exp Brain Res 85:621-624.

Kelso SR, Nelson TE, Leonard JP (1992) Protein kinase C-mediated cnhancement of NMDA currents by metabotropic glutamate receptors in Xenopus oocytes. J Physiol (Lond) 449:705-718.

Kimura F, Nishigori A, Shirokawa T, Tsumoto T (1989) Long-term potentiation and NMDA receptors in the visual cortex of young rats. J Physiol (Lond) 414:125-144.

Kirkwood A, Bear MF (1994) Hebbian synapses in visual cortex. J Neurosci, in press.
Kirkwood A, Aizenman CD, Bear MF (1992) Common forms of plasticity in hippocampus and visual cortex in vitro. Soc Neurosci Abstr 18:1499.

Kirkwood A, Dudek SD, Gold JT, Aizenman CD, Bear MF (1993) Common forms of synaptic plasticity in hippocampus and neocortex in vitro. Science 260:1518-1521.

Komatsu Y, Iwakiri M (1992) Low-threshold $\mathrm{Ca}^{2+}$ channels mediate induction of long-term potentiation in kitten visual cortex. J Neurophysiol 67:401-410.

Komatsu Y, Nakajima S, Toyama K (1991) Induction of long-term potentiation without the participation of $N$-methyl-D-aspartate receptors in kitten visual cortex. J Neurophysiol 65:20-32.

Lee KS (1982) Sustained enhancement of evoked potentials following brief, high-frequency stimulation of the cerebral cortex in vitro. Brain Res 239:617-623.

Lisman J (1989) A mechanism for the Hebb and the anti-Hebb processes underlying learning and memory. Proc Natl Acad Sci USA 86: 9574-9578.

Malenka RC, Kauer JA, Perkel DJ, Mauk MD, Kelly PT, Nicoll RA, Waxham MN (1989) An essential role for postsynaptic calmodulin and protein kinase activity in long-term potentiation. Nature 340 : 554-557.

Mallinow R, Schulman H, Tsien RW (1989) Inhibition of postsynaptic PKC or CaMKII blocks induction but not expression of LTP. Science 245:862-866.

Merzenich MM (1987) Dynamic neocortical processes and the origins of higher brain functions. In: The neural and molecular bases of learning (Changeux J-P, Konishi M, eds), pp 337-358. Chichester: Wiley.

Miller EK, Li L, Desimone R (1991) A neural mechanism for working and recognition memory in inferior temporal cortex. Science 254: $1377-1379$.

Mitzdorf U (1985) Current source-density method and application in cat cerebral cortex: investigation of evoked potentials and EEG phenomena. Physiol Rev 65:37-100.

Mulkey RM, Malenka RC (1992) Mechanisms underlying induction of homosynaptic long-term depression in area $\mathrm{CAl}$ of the hippocampus. Neuron 9:967-975.

Mulkey RM, Herron CE, Malenka RC (1993) An essential role for protein phosphatases in hippocampal long-term depression. Science, in press.

Wiesel TN (1982) Postnatal development of the visual cortex and the influence of the environment. Nature 299:583-592. 